\title{
Non-steroidal anti-inflammatory drugs and covid-19
}

\author{
Extra risk is plausible on current balance of evidence
}

\author{
Paul Little professor of primary care research
}

University of Southampton, Southampton, UK

Emerging evidence suggests that the most serious complications of covid-19 are sepsis and cardiovascular or respiratory complications. They occur predominantly in elderly people and those with underlying health conditions. ${ }^{1}$ Does use of non-steroidal anti-inflammatory drugs (NSAIDs) increase these risks? We don't know for certain, but additional risks are plausible on the current balance of evidence.

\section{Evidence of harm}

In observational studies, long term use of NSAIDs such as ibuprofen, naproxen, and diclofenac has been associated with higher rates of cardiovascular outcomes such as myocardial infarction, heart failure, and stroke ${ }^{2}$ - albeit with ongoing debate about residual confounding. ${ }^{3}$ Acute respiratory tract infections are already associated with increased risk of stroke and myocardial infarction, and short term use of NSAIDs during the illness is associated with further increases in risk. ${ }^{45}$ NSAIDs cause nephrotoxicity, ${ }^{67}$ which is more likely among the patient groups most likely to be seriously affected by covid-19 and is exacerbated by fever and dehydration.

A recent review of case-control studies suggests that NSAIDs are associated with higher rates of complications after respiratory tract infections, including complicated pneumonia, pleural effusions, prolonged illness, peritonsillar abscess, dissemination of infection to more than one site, or suppuration. ${ }^{8}$ NSAIDs were also associated with delays in the prescription of effective antibiotic treatment for patients requiring hospital admission. ${ }^{8}$

The review's authors say the findings are plausibly explained by the inhibiting effect of NSAIDs on cyclo-oxygenases, which reduces polymorphonuclear recruitment and also inhibits the synthesis of lipoxins and resolvins, delaying the resolution of inflammation. ${ }^{8}$ Case-control evidence is limited by confounding by indication, whereby NSAIDs are prescribed to treat the early symptoms of complications and are not causally related to those complications. But these associations persist even in studies that control for this kind of confounding. ${ }^{89}$ Furthermore, one large case-control study found an association between NSAIDs and respiratory complications, whether the NSAIDs were taken long term or just to treat a short acute illness. This suggests that the association is not simply a result of increased prescription in response to acute illness. ${ }^{10}$
What about trial evidence in primary care settings? A large trial $(\mathrm{n}=889)$ randomised patients presenting with respiratory tract infections to advice to take paracetamol, ibuprofen, or both. ${ }^{11}$ Re-consultations with new or unresolved symptoms or complications were documented in $12 \%$ of the paracetamol group and $20 \%$ of the ibuprofen group (adjusted risk ratio 1.67, 95\% confidence interval 1.12 to 2.38 ). The 11 complications recorded in the ibuprofen group were quinsy, sinusitis $(n=3)$, meningitis, pneumonia, otitis media $(n=3)$, and progression or non-resolution of otitis media $(\mathrm{n}=2)$.

A second randomised trial in 3044 primary care patients gave half access to a website advising on self-management of respiratory tract infections, including advice to use NSAIDs. ${ }^{12}$ Multivariate analyses suggested that among participants who developed respiratory tract infections, those with access to the website had more prolonged illness than controls without access - that is, more days of illness rated moderately bad or worse (difference 0.52 days; $95 \%$ CI 0.06 to $0.97, \mathrm{P}=0.026$ ). The effect could not be explained by reporting bias or confounding by indication and was attenuated after the authors controlled for use of the ibuprofen web pages.

This pragmatic trial evidence supports observational data suggesting that NSAIDs may cause more prolonged illness or complications when taken during respiratory tract infections.

\section{What about covid-19?}

Clearly, the big unknown is whether any of this evidence applies in the covid-19 epidemic. The evidence to date is not strong enough to support advising against all use of NSAIDs: the primary care trials above tested more regular dosing during respiratory infections, so we have little evidence about intermittent use, and it seems likely that that intermittent or occasional use could help patients with covid-19-for example, to relieve night time symptoms and aid sleep if paracetamol is inadequate, given the importance of sleep in immune defence. ${ }^{13}$ Furthermore, patients with covid-19 may need NSAIDs for other symptoms such as musculoskeletal pain.

People taking low dose aspirin for secondary prevention of cardiovascular disease should continue their treatment. Aspirin 
has anti-inflammatory effects only at much higher doses (eg, 1-4 g per day).

In summary, reasonable evidence exists of a link between NSAIDs and both respiratory and cardiovascular adverse effects in several settings, but so far we have no evidence relating specifically to people with covid-19. Pending further research, a pragmatic and cautionary approach would be for the public to avoid these plausible harms: regular NSAID use should probably not be recommended as the first line option for managing the symptoms of covid-19.

Competing interests: The BMJ has judged that there are no disqualifying financial ties to commercial companies. The author declares no other interests. The BMJ policy on financial interests is here: https://www.bmj.com/sites/default/files/ attachments/resources/2016/03/16-current-bmj-education-coi-form.pdf." Provenance and peer review: Commissioned; not externally peer reviewed.

1 Zhou F, Yu T, Du R, etal . Clinical course and risk factors for mortality of adult inpatients with COVID-19 in Wuhan, China: a retrospective cohort study. Lancet 2020;S0140-6736(20)30566-3. 10.1016/S0140-6736(20)30566-3 32171076

2 Bhala N, Emberson J, Merhi A, etal. Coxib and traditional NSAID Trialists' (CNT) Collaboration. Vascular and upper gastrointestinal effects of non-steroidal anti-inflammatory drugs: meta-analyses of individual participant data from randomised trials. Lancet 2013;382:769-79. 10.1016/S0140-6736(13)60900-9 23726390

3 Moore N. Coronary risks associated with diclofenac and other NSAIDs: an update. Drug Saf 2020. 10.1007/s40264-019-00900-8 31916080
4 Wen YC, Hsiao FY, Lin ZF, Fang CC, Shen LJ. Risk of stroke associated with use of nonsteroidal anti-inflammatory drugs during acute respiratory infection episode. Pharmacoepidemiol Drug Saf 2018;27:645-51. 10.1002/pds.4428 29659118

5 Wen YC, Hsiao FY, Chan KA, Lin ZF, Shen LJ, Fang CC. Acute respiratory infection and use of nonsteroidal anti-inflammatory drugs on risk of acute myocardial infarction: a nationwide case-crossover study. J Infect Dis 2017;215:503-9. 10.1093/infdis/jiw603 28158479

6 Clavé S, Rousset-Rouvière C, Daniel L, Tsimaratos M. The invisible threat of non-steroidal anti-inflammatory drugs for kidneys. Front Pediatr 2019;7:520. 10.3389/fped.2019.00520 31921731

7 Zhang X, Donnan PT, Bell S, Guthrie B. Non-steroidal anti-inflammatory drug induced acute kidney injury in the community dwelling general population and people with chronic kidney disease: systematic review and meta-analysis. BMC Nephrol 2017;18:256. 10.1186/s12882-017-0673-8 28764659

8 Voiriot G, Philippot Q, Elabbadi A, Elbim C, Chalumeau M, Fartoukh M. Risks related to the use of non-steroidal anti-inflammatory drugs in community-acquired pneumonia in adult and pediatric patients. J Clin Med 2019;8:E786. 10.3390/jcm8060786 31163625

9 Le Bourgeois M, Ferroni A, Leruez-Ville M, etal. Children, Antibiotics, Nonsteroidal Anti-inflammatory Drugs and Childhood Empyema (ChANCE) Study Group. Nonsteroidal anti-inflammatory drug without antibiotics for acute viral infection increases the empyema risk in children: a matched case-control study. J Pediatr 2016;175:47-53.e3 10.1016/j.jpeds.2016.05.025 27339249

10 Basille D, Thomsen RW, Madsen M, etal . Nonsteroidal antiinflammatory drug use and clinical outcomes of community-acquired pneumonia. Am J Respir Crit Care Med 2018;198:128-31. 10.1164/rccm.201802-0229LE 29461860

11 Little P, Moore M, Kelly J, etal. PIPS Investigators. Ibuprofen, paracetamol, and steam for patients with respiratory tract infections in primary care: pragmatic randomised factorial trial. BMJ 2013;347:f6041.. 10.1136/bmj.f6041 24162940

12 Little P, Stuart B, Andreou P, etal . Primary care randomised controlled trial of a tailored interactive website for the self-management of respiratory infections (internet doctor). BMJ Open 2016;6:e009769. 10.1136/bmjopen-2015-009769 27098821

13 Besedovsky L, Lange T, Haack M. The sleep-immune crosstalk in health and disease Physiol Rev 2019;99:1325-80. 10.1152/physrev.00010.2018 30920354

Published by the BMJ Publishing Group Limited. For permission to use (where not already granted under a licence) please go to http://group.bmj.com/group/rights-licensing/ permissions 